\title{
DIFFUSIONS WITH A NONLINEAR IRREGULAR DRIFT COEFFICIENT AND PROBABILISTIC INTERPRETATION OF GENERALIZED BURGERS' EQUATIONS
}

\author{
B. JOURDAIN
}

\begin{abstract}
We prove existence and uniqueness for two classes of martingale problems involving a nonlinear but bounded drift coefficient. In the first class, this coefficient depends on the time $t$, the position $x$ and the marginal of the solution at time $t$. In the second, it depends on $t, x$ and $p(t, x)$, the density of the time marginal w.r.t. Lebesgue measure. As far as the dependence on $t$ and $x$ is concerned, no continuity assumption is made. The results, first proved for the identity diffusion matrix, are extended to bounded, uniformly elliptic and Lipschitz continuous matrices. As an application, we show that within each class, a particular choice of the coefficients leads to a probabilistic interpretation of generalizations of Burgers' equation.
\end{abstract}

\section{INTRODUCTION}

In this paper, we are interested in diffusions given by two nonlinear martingale problems. Each problem is closely linked to the nonlinear partial differential equation satisfied by the time marginals of any solution. Under our assumptions on the diffusion and the drift coefficients, the time marginals are absolutely continuous (for $t>0$ ) and the partial differential equation provides a nice evolution equation for the densities. Our proofs for existence and uniqueness are based on fixed-point methods for this evolution equation.

The first section is devoted to a mean field martingale problem. For $F$ a bounded measurable $\mathbb{R}^{d}$ valued function on $[0,+\infty) \times \mathbb{R}^{d} \times \mathcal{P}\left(\mathbb{R}^{d}\right)$, Lipschitz continuous in its last variable for the total variation metric, we say that $P \in \mathcal{P}\left(C\left([0,+\infty), \mathbb{R}^{d}\right)\right)$ with time marginals $\left(P_{t}\right)_{t>0}$ solves the nonlinear martingale problem (MP1) starting at $m \in \mathcal{P}\left(\mathbb{R}^{d}\right)$ if $P_{0}=m$ and for any $\phi \in C_{b}^{2}\left(\mathbb{R}^{d}\right)$

$$
\phi\left(X_{t}\right)-\phi\left(X_{0}\right)-\int_{0}^{t}\left(\frac{1}{2} \Delta \phi\left(X_{s}\right)+F\left(s, X_{s}, P_{s}\right) \cdot \nabla \phi\left(X_{s}\right)\right) d s
$$

is a $P$-martingale where $X$ denotes the canonical process on $C\left([0,+\infty), \mathbb{R}^{d}\right)$. We prove existence and uniqueness for (MP1).

If the drift coefficient $F$ was Lipschitz continuous in its second and last

URL address of the journal: http://www.emath.fr/ps/

Received by the journal December 27, 1996. Revised July 9, 1997. Accepted for publication August 29, 1997.

(C) Société de Mathématiques Appliquées et Industrielles. Typeset by $\mathrm{IAT}_{\mathrm{EX}} \mathrm{X}$ 
variables for the sum of the Fortet-Mourier metric on $\mathcal{P}\left(\mathbb{R}^{d}\right)$

$$
\rho\left(\mu, \mu^{\prime}\right)=\sup \left\{\int \phi d \mu-\int \phi d \mu^{\prime} ;|\phi(x)-\phi(y)| \leq|x-y| \wedge 1\right\}
$$

and the Euclidian metric on $\mathbb{R}^{d}$, we could apply classical existence and uniqueness results for nonlinear diffusions, which are proved by samplepath couplings (see for example Graham (1992)). But our assumptions are much weaker since we do not suppose any continuity in the second variable and the Fortet-Mourier metric is obviously smaller than the total variation metric. The counterpart is that the diffusion coefficient is linear and the drift coefficient $F$ is bounded. By a fixed-point method, we prove that the evolution equation satisfied by the densities of the time marginals of any solution of (MP1) admits a unique solution. The results for the martingale problem itself follow quite immediately.

By our theorem, for $d=1$ and $F(s, x, \mu)=\left(\int_{\mathbb{R}} H(x-y) \mu(d y)\right)^{q}$ where $q \geq 1$ and $H$ denotes the Heaviside function $\left(H(x)=1_{\{x \geq 0\}}\right)$, the martingale problem (MP1) starting at $m$ admits a unique solution $P$. Let $V(t, x)$ and $v(x)$ be the distribution functions of $P_{t}$ and $m$. Generalizing results given by Bossy et al (1996) for Burgers' equation $(q=1)$, we prove that $V$ is a weak solution of

$$
\frac{\partial u}{\partial t}=\frac{1}{2} \frac{\partial^{2} u}{\partial x^{2}}-\frac{1}{q+1} \frac{\partial\left(u^{q+1}\right)}{\partial x}
$$

with initial condition $v$ and obtain $P$ as the propagation of chaos limit of a sequence of weakly interacting particle systems. Our propagation of chaos result is trajectorial and stronger than the one proved by Bossy and Talay.

The second section deals with a moderate martingale problem in which the drift coefficient depends on the densities of the time marginals. Thus the nonlinearity is more ticklish. For $F$ a bounded measurable $\mathbb{R}^{d}$ valued function on $[0,+\infty) \times \mathbb{R}^{d} \times \mathbb{R}$, satisfying

$$
\forall s \geq 0, \forall x \in \mathbb{R}^{d}, \forall y, y^{\prime} \in \mathbb{R},\left|y F(s, x, y)-y^{\prime} F\left(s, x, y^{\prime}\right)\right| \leq K_{F}\left|y-y^{\prime}\right|,
$$

we say that $P \in \mathcal{P}\left(C\left([0,+\infty), \mathbb{R}^{d}\right)\right)$ with time marginals $\left(P_{t}\right)_{t>0}$ absolutely continuous with respect to Lebesgue measure for $t>0$ solves the nonlinear martingale problem (MP2) starting at $m \in \mathcal{P}\left(\mathbb{R}^{d}\right)$ if $P_{0}=m$ and for any $\phi \in C_{b}^{2}\left(\mathbb{R}^{d}\right)$

$$
\phi\left(X_{t}\right)-\phi\left(X_{0}\right)-\int_{0}^{t}\left(\frac{1}{2} \Delta \phi\left(X_{s}\right)+F\left(s, X_{s}, p\left(s, X_{s}\right)\right) . \nabla \phi\left(X_{s}\right)\right) d s
$$

is a $P$-martingale where for any $t>0, p(t,$.$) is a density of P_{t}$.

We prove existence and uniqueness for (MP2). This generalizes a result given by Méléard et al (1987) for $F: \mathbb{R}^{d} \times \mathbb{R} \rightarrow \mathbb{R}^{d}$ bounded and satisfying a stronger Lipschitz continuity property: $\forall x, x^{\prime} \in \mathbb{R}^{d}, \forall y, y^{\prime} \in \mathbb{R}$,

$$
\left|F(x, y)-F\left(x^{\prime}, y^{\prime}\right)\right|+\left|y F(x, y)-y^{\prime} F\left(x^{\prime}, y^{\prime}\right)\right| \leq K_{F}\left(\left|x-x^{\prime}\right|+\left|y-y^{\prime}\right|\right) .
$$

They obtain existence for the corresponding martingale problem (MP2) as a consequence of a propagation of chaos result for a sequence of moderately interacting particle systems. As for 11 , we give a direct proof again based on a fixed-point method for the evolution equation satisfied by $p$. 
Thanks to this result, we show how it is possible to associate a probabilistic representation with some classical solutions of Burgers' equation, as it was sketched by Oelschläger (1985). The initial conditions concerned are bounded probability densities on $\mathbb{R}$.

In the last section we generalize the previous existence and uniqueness results to similar martingale problems with a Lipschitz continuous, bounded and uniformly elliptic diffusion coefficient.

Notations. Let $\Omega=C\left([0,+\infty), \mathbb{R}^{d}\right)$ endowed with the topology of uniform convergence on compact sets and with the corresponding Borel $\sigma$-field, $\Omega^{T}=$ $C\left([0, T], \mathbb{R}^{d}\right)$ endowed with the topology of uniform convergence, $X$ be the canonical process. For a Borel space $E, \mathcal{P}(E)$ is the space of probability measures on $E$ endowed with the topology of weak convergence. We also define the metric of total variation on $\mathcal{P}(E)$

$$
V\left(\mu, \mu^{\prime}\right)=\sup \left\{\int \phi d \mu-\int \phi d \mu^{\prime} ;\|\phi\|_{L^{\infty}(E)} \leq 1\right\} .
$$

If $Z$ is a random variable with values in $E$ let $\mathcal{L}(Z) \in \mathcal{P}(E)$ denote its law. If $P \in \mathcal{P}(\Omega),\left(P_{t}\right)_{t \geq 0}$ is the set of time marginals of $P$.

$$
\begin{aligned}
& \tilde{\mathcal{P}}(\Omega)=\left\{P \in \mathcal{P}(\Omega) ; \forall t>0, P_{t}\right. \text { is absolutely continuous } \\
& \text { with respect to Lebesgue measure\}. }
\end{aligned}
$$

If $P \in \tilde{\mathcal{P}}(\Omega)$, there is a measurable function $p(s, x)$ on $(0,+\infty) \times \mathbb{R}^{d}$ such that for any $s>0, p(s,$.$) is a density of P_{s}$ with respect to Lebesgue measure. See for example Meyer (1966) (pp. 193-194). Such a function is called a measurable version of the densities.

For $x \in \mathbb{R}^{d}$, let $|x|$ be the Euclidian norm of $x$.

For $t>0, G_{t}$ denotes the heat kernel on $\mathbb{R}^{d}: G_{t}(x)=(2 \pi t)^{-\frac{d}{2}} \exp \left(-\frac{|x|^{2}}{2 t}\right)$. The following estimate will be very useful:

$$
\text { for any } 1 \leq i \leq d, \quad\left\|\frac{\partial G_{t}}{\partial x_{i}}\right\|_{L^{1}} \leq \frac{1}{\sqrt{t}} .
$$

\section{THE MEAN FiELD MARTINGALE PROBLEM}

\subsection{Existence and Uniqueness}

Let $F$ be a measurable $\mathbb{R}^{d}$ valued function on $[0,+\infty) \times \mathbb{R}^{d} \times \mathcal{P}\left(\mathbb{R}^{d}\right)$ bounded by $M_{F}$ which satisfies the following Lipschitz continuity property

$$
\forall s \geq 0, \forall x \in \mathbb{R}^{d}, \forall \mu, \mu^{\prime} \in \mathcal{P}\left(\mathbb{R}^{d}\right),\left|F(s, x, \mu)-F\left(s, x, \mu^{\prime}\right)\right| \leq K_{F} V\left(\mu, \mu^{\prime}\right) .
$$

Definition 2.1. Let $m \in \mathcal{P}\left(\mathbb{R}^{d}\right)$. We say that $P \in \mathcal{P}(\Omega)$ with time marginals $\left(P_{t}\right)_{t \geq 0}$ solves the nonlinear martingale problem (MP1) starting at $m$ if $P_{0}=m$ and for any $\phi \in C_{b}^{2}\left(\mathbb{R}^{d}\right)$,

$$
\phi\left(X_{t}\right)-\phi\left(X_{0}\right)-\int_{0}^{t}\left(\frac{1}{2} \Delta \phi\left(X_{s}\right)+F\left(s, X_{s}, P_{s}\right) \cdot \nabla \phi\left(X_{s}\right)\right) d s
$$

is a $P$-martingale.

TheOREM 2.2. For any $m \in \mathcal{P}\left(\mathbb{R}^{d}\right)$, the nonlinear problem (MP1) starting at $m$ admits a unique solution. 
The following lemma gives an integral equation satisfied by any measurable version of the densities of the solution of a linear martingale problem. The proof of Theorem 2.2 which is based on this equation, is postponed after the proof of the lemma.

LEMMA 2.3. Let $m \in \mathcal{P}\left(\mathbb{R}^{d}\right), g$ be a measurable $\mathbb{R}^{d}$ valued function on $[0,+\infty) \times \mathbb{R}^{d}$ bounded by $M_{g}$ and $P$ be the unique solution of the martingale problem: $P_{0}=m$ and for any $\phi \in C_{b}^{2}\left(\mathbb{R}^{d}\right)$,

$\phi\left(X_{t}\right)-\phi\left(X_{0}\right)-\int_{0}^{t}\left(\frac{1}{2} \Delta \phi\left(X_{s}\right)+g\left(s, X_{s}\right) . \nabla \phi\left(X_{s}\right)\right) d s$ is a P-martingale.

Then $P \in \tilde{P}(\Omega)$. Any measurable version of the densities $p(s, x)$ satisfies the evolution equation,

$$
\forall t>0, p(t, x)=G_{t} * m(x)-\sum_{i=1}^{d} \int_{0}^{t} \frac{\partial G_{t-s}}{\partial x_{i}} *\left(\left(p g_{i}\right)(s, .)\right)(x) d s \text { a.e. }
$$

Moreover, if $q$ is a measurable function on $(0,+\infty) \times \mathbb{R}^{d}$ which satisfies (2.2) and

$$
\forall T>0, \sup _{t \in(0, T]}\|q(t, .)\|_{L^{1}}<+\infty
$$

then $q$ is a measurable version of the densities for $P$.

Proof. Existence and uniqueness for the martingale problem is a consequence of Girsanov's theorem. Let us prove that the solution $P$ belongs to $\tilde{P}(\Omega)$. Under $P$, by Paul Levy's characterization, $X_{t}-X_{0}-\int_{0}^{t} g\left(s, X_{s}\right) d s$ is a Brownian motion. We introduce the exponential martingale

$$
Z_{s}=\exp \left(-\int_{0}^{s} g\left(r, X_{r}\right) \cdot d X_{r}+\frac{1}{2} \int_{0}^{s}\left|g\left(r, X_{r}\right)\right|^{2} d r\right) .
$$

Let $t>0$. We set $Q=Z_{t} \times P$. By Girsanov's theorem, $\left(\beta_{s}=X_{s}-X_{0}\right)_{s \in[0, t]}$ is a Brownian motion under $Q$. Let $f$ be a continuous function with compact support in $\mathbb{R}^{d}$.

$$
\begin{aligned}
& \mathbb{E}\left(\left|f\left(X_{t}\right)\right|\right)=\mathbb{E}^{Q}\left(\frac{1}{Z_{t}}\left|f\left(X_{t}\right)\right|\right) \leq \sqrt{\mathbb{E}^{Q}\left(\frac{1}{Z_{t}^{2}}\right)} \sqrt{\mathbb{E}^{Q}\left(f^{2}\left(X_{t}\right)\right)} \\
& \mathbb{E}^{Q}\left(f^{2}\left(X_{t}\right)\right)=\int_{\mathbb{R}^{d}} f^{2}(x) G_{t} * m(x) d x \leq \frac{1}{(2 \pi t)^{\frac{d}{2}}}\|f\|_{L^{2}}^{2} \\
& \frac{1}{Z_{t}^{2}}=\exp \left(\int_{0}^{t} 2 g\left(s, X_{s}\right) \cdot d \beta_{s}-\frac{1}{2} \int_{0}^{t}\left|2 g\left(s, X_{s}\right)\right|^{2} d s+\int_{0}^{t}\left|g\left(s, X_{s}\right)\right|^{2} d s\right) .
\end{aligned}
$$

The last equation implies

$$
\mathbb{E}^{Q}\left(\frac{1}{Z_{t}^{2}}\right) \leq \exp \left(M_{g}^{2} t\right)
$$

With equations (2.3), (2.4) and (2.5), we conclude

$$
\left|\mathbb{E}\left(f\left(X_{t}\right)\right)\right| \leq \mathbb{E}\left(\left|f\left(X_{t}\right)\right|\right) \leq \frac{1}{(2 \pi t)^{\frac{d}{4}}} \exp \left(\frac{M_{g}^{2} t}{2}\right)\|f\|_{L^{2}} .
$$

Hence $P_{t}$ is absolutely continuous with respect to Lebesgue measure and $P \in \tilde{\mathcal{P}}(\Omega)$. 
Let $p(s, x)$ be a measurable version of the densities for $P, \psi$ be a $C^{2}$ function with compact support in $\mathbb{R}^{d}$ and $t>0$. We set $\phi(s, x)=G_{t-s} * \psi(x)$ for $s \in[0, t)$ and $\phi(t, x)=\psi(x)$. The function $\phi$ belongs to $C_{b}^{1,2}\left([0, t] \times \mathbb{R}^{d}\right)$ and satisfies

$$
\forall(s, x) \in[0, t] \times \mathbb{R}^{d}, \frac{\partial \phi}{\partial s}(s, x)+\frac{1}{2} \Delta \phi(s, x)=0 .
$$

Since $X_{t}-X_{0}-\int_{0}^{t} g\left(s, X_{s}\right) d s$ is a $P$-Brownian motion, Itô's formula implies

$$
\mathbb{E}\left(\phi\left(t, X_{t}\right)\right)=\mathbb{E}\left(\phi\left(0, X_{0}\right)+\int_{0}^{t}\left(\frac{\partial \phi}{\partial s}+\frac{1}{2} \Delta \phi+g . \nabla \phi\right)\left(s, X_{s}\right) d s\right) .
$$

By (2.7), we get rid of $\frac{\partial \phi}{\partial s}+\frac{1}{2} \Delta \phi$. Applying Fubini's theorem, we obtain

$$
\begin{aligned}
\int_{\mathbb{R}^{d}} \psi(x) p(t, x) d x & =\int_{\mathbb{R}^{d}} G_{t} * \psi(x) m(d x) \\
& +\int_{(0, t] \times \mathbb{R}^{d}} \sum_{i=1}^{d} \int_{\mathbb{R}^{d}} \frac{\partial G_{t-s}}{\partial x_{i}}(x-y) \psi(y) d y\left(g_{i} p\right)(s, x) d x d s \\
& =\int_{\mathbb{R}^{d}} \psi(x) G_{t} * m(x) d x \\
& -\int_{\mathbb{R}^{d}} \sum_{i=1}^{d} \int_{0}^{t} \frac{\partial G_{t-s}}{\partial x_{i}} *\left(\left(g_{i} p\right)(s, .)\right)(y) d s \psi(y) d y .
\end{aligned}
$$

Hence $p$ satisfies (2.2).

To conclude the proof, we consider $q$ satisfying (2.2) and

$$
\begin{gathered}
\forall T>0, \sup _{t \in(0, T]}\|q(t, .)\|_{L^{1}}<+\infty \\
\|p(t, .)-q(t, .)\|_{L^{1}} \leq \sum_{i=1}^{d} \int_{0}^{t}\left\|\frac{\partial G_{t-s}}{\partial x_{i}}\right\|_{L^{1}}\left\|g_{i}(s, .)(p(s, .)-q(s, .))\right\|_{L^{1}} d s \\
\leq M_{g} \sqrt{d} \int_{0}^{t} \frac{\|p(s, .)-q(s, .)\|_{L^{1}}}{\sqrt{t-s}} d s .
\end{gathered}
$$

After an iteration, we get

$$
\begin{aligned}
\|p(t, .)-q(t, .)\|_{L^{1}} & \leq M_{g}^{2} d \int_{0}^{t} \frac{1}{\sqrt{t-s}} \int_{0}^{s} \frac{\|p(r, .)-q(r, .)\|_{L^{1}}}{\sqrt{s-r}} d r d s \\
& \leq M_{g}^{2} d \int_{0}^{t}\|p(r, .)-q(r, .)\|_{L^{1}} \int_{r}^{t} \frac{1}{\sqrt{t-s} \sqrt{s-r}} d s d r \\
& \leq \pi M_{g}^{2} d \int_{0}^{t}\|p(r, .)-q(r, .)\|_{L^{1}} d r .
\end{aligned}
$$

Gronwall's lemma implies $\forall t>0,\|p(t, .)-q(t, .)\|_{L^{1}}=0$ which proves that $q$ is a measurable version of the densities for $P$.

We are now ready to show Theorem 2.2.

Proof. The key idea is the following. If $(Q(t))_{t>0} \in C\left([0,+\infty), \mathcal{P}\left(\mathbb{R}^{d}\right)\right)$, by Girsanov's theorem, the martingale problem in which the nonlinearity $P_{s}$ in (2.1) is replaced by $Q(s)$ admits a unique solution $P^{Q}$. We consider the 
correspondence between $(Q(t))_{t \geq 0}$ and the time marginals $\left(P_{t}^{Q}\right)_{t \geq 0}$ of the solution. If $P$ solves the nonlinear problem (MP1), then $\left(P_{t}\right)_{t \geq 0}$ is a fixedpoint of this map. Conversely, if $(Q(t))_{t>0}$ is a fixed-point, then $P^{Q}$ solves the nonlinear problem (MP1).

Let $T>0$. We define

$$
\begin{aligned}
A_{m, T}=\left\{Q \in C\left([0, T], \mathcal{P}\left(\mathbb{R}^{d}\right)\right) ; Q(0)=m \text { and } \forall t \in(0, T], Q(t)\right. \\
\quad \text { is absolutely continuous with respect to Lebesgue measure }\} .
\end{aligned}
$$

If $Q \in A_{m, T}$, let $\lambda(Q)$ denote a measurable version of the densities for $Q$. $A_{m, T}$ is complete for the metric

$$
D\left(Q, Q^{\prime}\right)=\sup _{t \in(0, T]} V\left(Q(t), Q^{\prime}(t)\right)=\sup _{t \in(0, T]}\left\|\lambda(Q)(t)-\lambda\left(Q^{\prime}\right)(t)\right\|_{L^{1}}
$$

Let $t_{0} \geq 0$. For $Q \in A_{m, T}$ we define $\left(\psi_{t_{0}, m}(Q)(t)\right)_{t \in[0, T]}$ as the time marginals of the unique solution of the martingale problem:

$$
\left\{\begin{array}{l}
P \in \mathcal{P}\left(\Omega^{T}\right), P_{0}=m \text { and } \forall \phi \in C_{b}^{2}\left(\mathbb{R}^{d}\right), \\
\phi\left(X_{t}\right)-\phi\left(X_{0}\right)-\int_{0}^{t}\left(\frac{1}{2} \Delta \phi\left(X_{s}\right)+F\left(t_{0}+s, X_{s}, Q_{s}\right) \cdot \nabla \phi\left(X_{s}\right)\right) d s \\
\text { is a } P \text {-martingale. }
\end{array}\right.
$$

Lemma 2.3 implies that for any $t \in(0, T], \psi_{t_{0}, m}(Q)(t)$ is absolutely continuous with respect to Lebesgue measure. Hence $\psi_{t_{0}, m}(Q) \in A_{m, T}$. We are going to prove that if $T$ is small enough, $\psi_{t_{0}, m}$ is a contraction on $A_{m, T}$. Using equation (2.2) given by Lemma 2.3, we obtain for $Q, Q^{\prime} \in A_{m, T}$ and $t \in(0, T]$,

$$
\begin{aligned}
& \left\|\lambda\left(\psi_{t_{0}, m}(Q)\right)(t)-\lambda\left(\psi_{t_{0}, m}\left(Q^{\prime}\right)\right)(t)\right\|_{L^{1}} \\
& \leq \sum_{i=1}^{d} \int_{0}^{t}\left\|\frac{\partial G_{t-s}}{\partial x_{i}}\right\|_{L^{1}} \| \lambda\left(\psi_{t_{0}, m}(Q)\right)(s) F_{i}\left(t_{0}+s, ., Q(s)\right) \\
& -\lambda\left(\psi_{t_{0}, m}\left(Q^{\prime}\right)\right)(s) F_{i}\left(t_{0}+s, ., Q^{\prime}(s)\right) \|_{L^{1}} d s \\
& \leq \int_{0}^{t}\left(\left\|\lambda\left(\psi_{t_{0}, m}(Q)\right)(s)\right\|_{L^{1}}\left\|\sum_{i=1}^{d} \mid F_{i}\left(t_{0}+s, ., Q(s)\right)-F_{i}\left(t_{0}+s, ., Q^{\prime}(s)\right)\right\| \|_{L^{\infty}}\right. \\
& +\left\|\lambda\left(\psi_{t_{0}, m}(Q)\right)(s)-\lambda\left(\psi_{t_{0}, m}\left(Q^{\prime}\right)\right)(s)\right\|_{L^{1}} \times \\
& \left.\left\|\sum_{i=1}^{d}\left|F_{i}\left(t_{0}+s, ., Q^{\prime}(s)\right)\right|\right\|_{L^{\infty}}\right) \frac{d s}{\sqrt{t-s}} \\
& \leq 2 \sqrt{d T}\left(K_{F} D\left(Q, Q^{\prime}\right)+M_{F} D\left(\psi_{t_{0}, m}(Q), \psi_{t_{0}, m}\left(Q^{\prime}\right)\right)\right)
\end{aligned}
$$

Hence

$$
\left(1-2 \sqrt{d T} M_{F}\right) D\left(\psi_{t_{0}, m}(Q), \psi_{t_{0}, m}\left(Q^{\prime}\right)\right) \leq 2 \sqrt{d T} K_{F} D\left(Q, Q^{\prime}\right) .
$$

We set $T=\frac{1}{4 d\left(M_{F}+2 K_{F}\right)^{2}}$. Then $D\left(\psi_{t_{0}, m}(Q), \psi_{t_{0}, m}\left(Q^{\prime}\right)\right) \leq \frac{1}{2} D\left(Q, Q^{\prime}\right)$. Picard's fixed-point theorem implies that $\psi_{t_{0}, m}$ admits a unique fixed-point in $A_{m, T}$.

Existence for the martingale problem (MP1). Let $Q^{0}$ denote the fixed-point of $\psi_{0, m}$ in $A_{m, T}$. If $Q^{n}$ is constructed, let $Q^{n+1}$ be the fixed-point of $\psi_{(n+1) T, Q^{n}(T)}$ in $A_{Q^{n}(T), T}$.

We set $Q(t)=Q^{n}(t-n T)$ if $t \in[n T,(n+1) T)$. Let $P$ be the solution of the 
martingale problem in which the nonlinearity in (2.1) is replaced by $Q(s)$. For any $\phi \in C_{b}^{2}\left(\mathbb{R}^{d}\right)$,

$$
\begin{aligned}
\phi\left(X_{n T+t}\right)-\phi\left(X_{n T}\right)-\int_{0}^{t}( & \left.F\left(n T+s, X_{n T+s}, Q^{n}(s)\right)\right) . \nabla \phi\left(X_{n T+s}\right) \\
& \left.+\frac{1}{2} \Delta \phi\left(X_{n T+s}\right)\right) d s \text { is a } P \text {-martingale. }
\end{aligned}
$$

Hence, by induction, $\forall n \in \mathbb{N}, \forall t \in[0, T], P_{n T+t}=Q^{n}(t)=Q(n T+t)$. And $P$ solves the problem (MP1).

Uniqueness for the martingale problem (MP1). If $P$ is a solution, Lemma 2.3 implies that for any $t>0, P_{t}$ is absolutely continuous with respect to Lebesgue measure. For any $n \in \mathbb{N},\left(P_{n T+t}\right)_{t \in[0, T]}$ is the fixedpoint of $\psi_{n T, P_{n T}}$ in $A_{P_{n T}, T}$. By induction, uniqueness for the fixed-points implies uniqueness for the time marginals $\left(P_{t}\right)_{t \geq 0}$. Since the nonlinearity in the definition of (MP1) is limited to the dependence of the drift coefficient on the time marginals, uniqueness for this problem follows immediately.

\subsection{Application}

Theorem 2.2 implies existence and uniqueness for martingale problems associated with a class of partial differential equations which includes Burgers' equation.

We set $q \geq 1, m \in \mathcal{P}(\mathbb{R})$. Let $f:(x, \mu) \in \mathbb{R} \times \mathcal{P}(\mathbb{R}) \rightarrow\left(\int_{\mathbb{R}} H(x-y) \mu(d y)\right)^{q}$ where $H(x)=1_{\{x \geq 0\}}$. As $f$ is the pointwise limit of the continuous functions $(x, \mu) \rightarrow\left(\int_{\mathbb{R}} H_{n}(x-y) \mu(d y)\right)^{q}$ with

$$
H_{n}(x)=n(x+1 / n) 1_{\{-1 / n \leq x \leq 0\}}+1_{\{x>0\}},
$$

this function is measurable. Moreover, since $f$ takes its values in $[0,1]$,

$$
\begin{aligned}
\left|f(x, \mu)-f\left(x, \mu^{\prime}\right)\right| & \leq q\left|\int_{\mathbb{R}} H(x-y) \mu(d y)-\int_{\mathbb{R}} H(x-y) \mu^{\prime}(d y)\right| \\
& \leq q V\left(\mu, \mu^{\prime}\right) .
\end{aligned}
$$

By Theorem 2.2, the martingale problem (MP1) corresponding to the particular choice $F(s, x, \mu)=f(x, \mu)$ admits a unique solution $P$ starting at $m$. Let $V(t, x)$ and $v(x)$ be the distribution functions of $P_{t}$ and $m$.

Bossy et al (1996) deal with the case $q=1$. They prove that $V$ is a weak solution of Burgers' equation

$$
\frac{\partial u}{\partial t}=\frac{1}{2} \frac{\partial^{2} u}{\partial x^{2}}-\frac{1}{2} \frac{\partial\left(u^{2}\right)}{\partial x}
$$

with initial condition $v$ and obtain $P$ as the propagation of chaos limit of a sequence of weakly interacting particle systems. Indeed they define $\left(X^{1, n}, \ldots, X^{n, n}\right)$ as the unique weak solution of the stochastic differential equation

$$
X_{t}^{i, n}=X_{0}^{i, n}+B_{t}^{i, n}+\int_{0}^{t} \frac{1}{n} \sum_{j=1}^{n} H\left(X_{s}^{i, n}-X_{s}^{j, n}\right) d s, 1 \leq i \leq n,
$$

where $\mathcal{L}\left(\left(X_{0}^{1, n}, \ldots, X_{0}^{n, n}\right)\right)=m^{\otimes n}$ and $\left(B^{1, n}, \ldots, B^{n, n}\right)$ is a $\mathbb{R}^{n}$-valued Brownian motion. They prove that for any $k \in \mathbb{N}^{*}, \mathcal{L}\left(\left(X^{1, n}, \ldots, X^{k, n}\right)\right)$ converges 
weakly to $P^{\otimes k}$ when $n \rightarrow+\infty$.

We generalize their results to any $q \geq 1$ in Proposition 2.4. In fact, we follow the idea of Méléard et al (1987) and prove a trajectorial propagation of chaos result. To obtain this result, we introduce a coupling between the particle systems and the limit processes with law $P$ that we define on the same probability space. Let $B^{i}, i \in \mathbb{N}^{*}$ be a sequence of independent $\mathbb{R}$-valued Brownian motions and $X_{0}^{i}, i \in \mathbb{N}^{*}$ be a sequence of random variables IID with law $m$ independent of the Brownian motions. According to Karatzas et al (1988) (Proposition 5.17 p.341), the one-dimensional stochastic differential equation

$$
Y_{t}^{i}=X_{0}^{i}+B_{t}^{i}+\int_{0}^{t}\left(H * P_{s}\left(Y_{s}^{i}\right)\right)^{q} d s
$$

admits a unique strong solution. Moreover, considering the linear martingale problem associated with this equation, by the existence part of the proof of Theorem 2.2, we obtain that the law of the solution is $P$. The process $Y_{i}$ is nonlinear in the following sense: the drift coefficient of the stochastic differential equation that it satisfies depends on the time marginals of its law.

Unlike in the one-dimensional case, to obtain a strong solution for a $n$ dimensional stochastic differential equation with $n>1$, it is necessary to assume that the coefficients are locally Lipschitz continuous. That is why we replace $H$ by $H_{n}\left(H_{n}(x)=n(x+1 / n) 1_{\{-1 / n \leq x \leq 0\}}+1_{\{x>0\}}\right)$ and define the weakly interacting particle system as the unique strong solution of the stochastic differential equation

$$
X_{t}^{i, n}=X_{0}^{i}+B_{t}^{i}+\int_{0}^{t}\left(\frac{1}{n} \sum_{j=1}^{n} H_{n}\left(X_{s}^{i, n}-X_{s}^{j, n}\right)\right)^{q} d s, 1 \leq i \leq n .
$$

Proposition 2.4. For any $q \geq 1$,

(i) The function $V$ is a weak solution of the generalized Burgers' equation

$$
\frac{\partial u}{\partial t}=\frac{1}{2} \frac{\partial^{2} u}{\partial x^{2}}-\frac{1}{q+1} \frac{\partial\left(u^{q+1}\right)}{\partial x} \text { with initial condition } v .
$$

(ii) If $\hat{P}$ denotes the image of $P$ by the mapping $X \in \Omega \rightarrow(X, X) \in \Omega^{2}$, for any $k \in \mathbb{N}^{*}, \mathcal{L}\left(\left(\left(X^{1, n}, Y^{1}\right), \ldots,\left(X^{k, n}, Y^{k}\right)\right)\right)$ converges weakly to $\hat{P}^{\otimes k}$ as $n$ goes to $+\infty$.

To understand the trajectorial nature of the propagation of chaos result (ii), remark for instance that, unlike the classical result: $\forall k \in \mathbb{N}^{*}$, $\mathcal{L}\left(\left(X^{1, n}, \ldots, X^{k, n}\right)\right)$ converges weakly to $P^{\otimes k}$, it implies:

$$
\forall T>0, \lim _{n \rightarrow+\infty} \mathbb{E}\left(1 \wedge \sup _{0 \leq t \leq T}\left|\left(X_{t}^{1, n}, \ldots, X_{t}^{k, n}\right)-\left(Y_{t}^{1}, \ldots, Y_{t}^{n}\right)\right|\right)=0 .
$$

Proof. (i) Our proof is a generalization of the one given by Bossy et al (1996). Under $P$, by Paul Levy's characterization, $X_{t}-X_{0}-\int_{0}^{t} V^{q}\left(s, X_{s}\right) d s$ is a Brownian motion. Let $p$ be a measurable version of the densities for $P$ and $\phi \in \mathcal{D}((0,+\infty) \times \mathbb{R})$. Applying Itô's formula and taking expectations, we get

$$
\int_{0}^{+\infty} \int_{\mathbb{R}} p(t, x)\left(\frac{\partial \phi}{\partial t}(t, x)+\frac{1}{2} \frac{\partial^{2} \phi}{\partial x^{2}}(t, x)+\frac{\partial \phi}{\partial x}(t, x) V^{q}(t, x)\right) d x d t=0 .
$$


Hence $p$ is a solution in $\mathcal{D}^{\prime}((0,+\infty) \times \mathbb{R})$ of the equation $\frac{\partial p}{\partial t}=\frac{1}{2} \frac{\partial^{2} p}{\partial x^{2}}-$ $\frac{\partial}{\partial x}\left(p V^{q}\right)$. Clearly, $\frac{\partial V}{\partial x}=p$ in $\mathcal{D}^{\prime}((0,+\infty) \times \mathbb{R})$. Moreover, approximating $p(t,$.$) in L^{1}(\mathbb{R})$ by continuous functions, we obtain that the distribution function of the bounded measure $p(t, x) V^{q}(t, x) d x$ is $\frac{1}{q+1} V^{q+1}(t, x)$. Hence

$$
\frac{\partial}{\partial x}\left(\frac{\partial V}{\partial t}-\frac{1}{2} \frac{\partial^{2} V}{\partial x^{2}}+\frac{1}{q+1} \frac{\partial\left(V^{q+1}\right)}{\partial x}\right)=0
$$

The spatial derivative of the distribution $\frac{\partial V}{\partial t}-\frac{1}{2} \frac{\partial^{2} V}{\partial x^{2}}+\frac{1}{q+1} \frac{\partial\left(V^{q+1}\right)}{\partial x}$ is zero, which implies that the distribution is invariant by translation. If $\phi \in \mathcal{D}((0,+\infty) \times \mathbb{R})$ and $z \rightarrow+\infty$,

$$
\int_{(0,+\infty) \times \mathbb{R}} V(t, x-z)\left(\frac{\partial \phi}{\partial t}(t, x)+\frac{1}{2} \frac{\partial^{2} \phi}{\partial x^{2}}(t, x)+\frac{V^{q}(t, x-z)}{q+1} \frac{\partial \phi}{\partial x}(t, x)\right) d x d t
$$

goes to 0 by Lebesgue's theorem. Therefore for any $\phi \in \mathcal{D}((0,+\infty) \times \mathbb{R})$,

$$
\int_{(0,+\infty) \times \mathbb{R}} V(t, x)\left(\frac{\partial \phi}{\partial t}(t, x)+\frac{1}{2} \frac{\partial^{2} \phi}{\partial x^{2}}(t, x)+\frac{V^{q}(t, x)}{q+1} \frac{\partial \phi}{\partial x}(t, x)\right) d x d t=0 .
$$

We conclude by proving that the initial condition is $v$. By density, equation (2.8) still holds if $\phi$ is $C^{1,2}$ with compact support in $(0,+\infty) \times \mathbb{R}$.

Let $\psi$ be $C^{1,2}$ with compact support in $[0,+\infty) \times \mathbb{R}$. For $n \in \mathbb{N}^{*}$, we introduce the $C^{1}$ functions

$$
g_{n}(s)=\left\{\begin{array}{l}
0 \text { if } s \in\left[0, \frac{1}{2 n}\right], \\
12 n^{2}\left(s-\frac{1}{2 n}\right)^{2}-16 n^{3}\left(s-\frac{1}{2 n}\right)^{3} \text { if } s \in\left[\frac{1}{2 n}, \frac{1}{n}\right], \\
1 \text { if } s \geq \frac{1}{n} .
\end{array}\right.
$$

The function $\phi_{n}=g_{n} \psi$ is $C^{1,2}$ with compact support in $(0,+\infty) \times \mathbb{R}$. Using (2.8) for $\phi_{n}$ we get

$$
\begin{aligned}
\int_{(0,+\infty) \times \mathbb{R}} & \left(\frac{\partial \psi}{\partial t}+\frac{1}{2} \frac{\partial^{2} \psi}{\partial x^{2}}+\frac{V^{q}}{q+1} \frac{\partial \psi}{\partial x}\right)(t, x) V(t, x) d t d x \\
= & \int_{\left(0, \frac{1}{n}\right] \times \mathbb{R}}\left(1-g_{n}(t)\right)\left(\frac{\partial \psi}{\partial t}+\frac{1}{2} \frac{\partial^{2} \psi}{\partial x^{2}}+\frac{V^{q}}{q+1} \frac{\partial \psi}{\partial x}\right)(t, x) V(t, x) d t d x \\
& -\int_{\left(0, \frac{1}{n}\right] \times \mathbb{R}} \frac{d g_{n}}{d t}(t) \psi(t, x) V(t, x) d t d x
\end{aligned}
$$

Since $P \in \mathcal{P}(\Omega)$, the map $t \rightarrow P_{t}$ is continuous and $\lim _{t \rightarrow 0} V(t, x)=v(x)$ for any $x$ such that $v$ is continuous at $x$. Hence by Lebesgue's theorem,

$$
\lim _{t \rightarrow 0} \int_{\mathbb{R}} \psi(t, x) V(t, x) d x=\int_{\mathbb{R}} \psi(0, x) v(x) d x .
$$

When $n \rightarrow+\infty$ in (2.9), we get

$$
\begin{gathered}
\int_{(0,+\infty) \times \mathbb{R}}\left(\frac{\partial \psi}{\partial t}+\frac{1}{2} \frac{\partial^{2} \psi}{\partial x^{2}}+\frac{V^{q}}{q+1} \frac{\partial \psi}{\partial x}\right)(t, x) V(t, x) d t d x \\
=-\int_{\mathbb{R}} \psi(0, x) v(x) d x .
\end{gathered}
$$


Therefore $V$ is a weak solution of the generalized Burgers' equation with initial condition $v$.

(ii) We now prove the propagation of chaos result. In the sequel, $\nu$ and $(X, Y)$ denote the canonical variables on $\mathcal{P}\left(\Omega^{2}\right)$ and $\Omega^{2}$. We set $\bar{\nu}_{r}=\nu \circ X_{r}^{-1}$. The couples $\left(X^{i, n}, Y^{i}\right), 1 \leq i \leq n$ are exchangeable. Therefore the propagation of chaos result is equivalent to the convergence in distribution of the empirical measures $\mu_{n}=\frac{1}{n} \sum_{i=1}^{n} \delta_{\left(X^{i, n}, Y^{i}\right)}$ considered as $\mathcal{P}\left(\Omega^{2}\right)$-valued random variables to $\delta_{\hat{P}}$ (see Sznitman (1991) and the references cited in it). Let $\pi_{n}$ denote the law of $\mu_{n}$.

According to Sznitman (1991), since the variables $\left(X^{i, n}, Y^{i}\right)$ are exchangeable, the tightness of the sequence $\left(\pi_{n}\right)_{n}$ is equivalent to the tightness of $\left(\mathcal{L}\left(X^{1, n}, Y^{1}\right)\right)_{n}$ which is equivalent to the tightness of $\left(\mathcal{L}\left(X^{1, n}\right)\right)_{n}$. These probability measures are tight since for any $T>0$ their images by the canonical restriction from $\Omega$ to $\Omega^{T}$ are tight (the drift coefficient is bounded by 1 uniformly in $t$ and $n$ ).

Let $\pi_{\infty}$ denote the limit of a convergent subsequence of $\left(\pi_{n}\right)_{n}$ that we still index by $n$ for simplicity. To prove that $\pi_{\infty}=\delta_{\hat{P}}$, we set $p \in \mathbb{N}^{*}$, $0 \leq s_{1} \leq s_{2} \leq \ldots \leq s_{p} \leq s \leq t, \phi \in C_{b}^{2}\left(\mathbb{R}^{2}\right), g \in C_{b}\left(\mathbb{R}^{2 p}\right)$, and define $G(\nu)$ to be equal to

$$
\begin{gathered}
<\nu,\left(\phi\left(X_{t}, Y_{t}\right)-\phi\left(X_{s}, Y_{s}\right)-\int_{s}^{t} \frac{1}{2}\left(\frac{\partial^{2} \phi}{\partial x^{2}}+2 \frac{\partial^{2} \phi}{\partial x \partial y}+\frac{\partial^{2} \phi}{\partial y^{2}}\right)\left(X_{r}, Y_{r}\right) d r\right. \\
\left.-\int_{s}^{t}\left(\frac{\partial \phi}{\partial x}\left(X_{r}, Y_{r}\right)\left(H * \bar{\nu}_{r}\left(X_{r}\right)\right)^{q}+\frac{\partial \phi}{\partial y}\left(X_{r}, Y_{r}\right)\left(H * P_{r}\left(Y_{r}\right)\right)^{q}\right) d r\right) \\
g\left(X_{s_{1}}, Y_{s_{1}}, \ldots, X_{s_{p}}, Y_{s_{p}}\right)>
\end{gathered}
$$

For $k \in \mathbb{N}^{*}$, we define $G_{k}(\nu)$ like $G$ with $H_{k}$ replacing $H$ in $\left(H * \bar{\nu}_{r}\left(X_{r}\right)\right)^{q}$ but not in $\left(H * P_{r}\left(Y_{r}\right)\right)^{q}$. If $\nu^{n} \rightarrow \nu$, the weak convergence of $\bar{\nu}_{r}$ to $\bar{\nu}_{r}$ implies that $H_{k} * \bar{\nu}_{r}(x)$ converges to $H_{k} * \bar{\nu}_{r}(x)$ uniformly for $x \in \mathbb{R}$. Moreover, for any $r>0, P_{r}$ is absolutely continuous with respect to Lebesgue measure and $y \rightarrow H * P_{r}(y)$ is continuous. Hence $G_{k}$ is continuous.

We are going to prove that $\mathbb{E}^{\pi_{\infty}}\left(G^{2}(\nu)\right)=0$. By the continuity and boundedness of $G_{k}$, we have

$$
\begin{gathered}
\mathbb{E}^{\pi_{\infty}}\left(G^{2}(\nu)\right) \leq 2 \limsup _{k \rightarrow+\infty}\left(\mathbb{E}^{\pi_{\infty}}\left(\left(G-G_{k}\right)^{2}(\nu)\right)+\lim _{n \rightarrow+\infty} \mathbb{E}\left(G_{k}^{2}\left(\mu^{n}\right)\right)\right) \\
\leq 2 \limsup _{k \rightarrow+\infty} \mathbb{E}^{\pi_{\infty}}\left(\left(G-G_{k}\right)^{2}(\nu)\right)+4 \limsup _{n \rightarrow+\infty} \mathbb{E}\left(G_{n}^{2}\left(\mu^{n}\right)\right) \\
+4 \limsup \limsup \mathbb{E}\left(\left(G_{k}-G_{n}\right)^{2}\left(\mu^{n}\right)\right) .
\end{gathered}
$$

Let us show that each term of the right-hand-side of $(2.10)$ is equal to 0 .

For the first term, it is a consequence of the convergence of $\left|H-H_{k}\right| * \bar{\nu}_{r}(x)$ to 0 for any $\nu \in \mathcal{P}\left(\Omega^{2}\right), x \in \mathbb{R}$ and $r \geq 0$ as $k \rightarrow+\infty$. Indeed, by the boundedness of $G, G_{k}, g$ and $\frac{\partial \phi}{\partial x}$ and the Lipschitz continuity of $x \rightarrow x^{q}$ for $0 \leq x \leq 1$, we have

$$
\begin{aligned}
\mathbb{E}^{\pi_{\infty}}\left(\left(G-G_{k}\right)^{2}(\nu)\right) & \leq C \mathbb{E}^{\pi_{\infty}}\left|G(\nu)-G_{k}(\nu)\right| \\
& \leq C \mathbb{E}^{\pi_{\infty}}\left(<\nu, \int_{s}^{t}\left|H-H_{k}\right| * \bar{\nu}_{r}\left(X_{r}\right) d r>\right)
\end{aligned}
$$


The second term is easy to deal with. Applying Itô's formula, we get

$$
G_{n}\left(\mu^{n}\right)=\frac{1}{n} \sum_{i=1}^{n} g\left(X_{s_{1}}^{i, n}, Y_{s_{1}}^{i}, \ldots, X_{s_{p}}^{i, n}, Y_{s_{p}}^{i}\right) \int_{s}^{t}\left(\frac{\partial \phi}{\partial x}+\frac{\partial \phi}{\partial y}\right)\left(X_{r}^{i, n}, Y_{r}^{i}\right) d B_{r}^{i}
$$

Hence $\mathbb{E}\left(G_{n}^{2}\left(\mu^{n}\right)\right) \leq C / n$ and we conclude $\lim _{n \rightarrow+\infty} \mathbb{E}\left(G_{n}^{2}\left(\mu^{n}\right)\right)=0$.

The third term is the most ticklish. By a calculation similar to the one carried out for the first term, we get

$$
\mathbb{E}\left(\left(G_{k}-G_{n}\right)^{2}\left(\mu^{n}\right)\right) \leq C \mathbb{E}\left(<\mu^{n}, \int_{s}^{t}\left|H_{n}-H_{k}\right| * \bar{\mu}_{r}^{n}\left(X_{r}\right) d r>\right)
$$

Hence if $(X, Y, Z, W)$ denotes the canonical variable on $\Omega^{4}$,

$$
\mathbb{E}\left(\left(G_{k}-G_{n}\right)^{2}\left(\mu^{n}\right)\right) \leq C \mathbb{E}\left(<\mu^{n} \otimes \mu^{n}, \int_{s}^{t} 1_{\left\{\left|X_{r}-Z_{r}\right| \leq \frac{1}{n \wedge k}\right\}} d r>\right) .
$$

By the exchangeability of the couples $\left(X^{i, n}, Y^{i}\right), 1 \leq i \leq n$,

$$
\begin{aligned}
\limsup _{n \rightarrow+\infty} \mathbb{E}\left(<\mu^{n} \otimes \mu^{n}, \int_{s}^{t}\right. & \left.1_{\left\{\left|X_{r}-Z_{r}\right| \leq \frac{1}{n \wedge k}\right\}} d r>\right) \\
= & \limsup _{n \rightarrow+\infty} \mathbb{E}\left(\int_{s}^{t} 1_{\left\{\left|X_{r}^{1, n}-X_{r}^{2, n}\right| \leq \frac{1}{n \wedge k}\right\}} d r\right) \\
\leq & \limsup _{n \rightarrow+\infty} \mathbb{E}\left(\int_{s}^{t} 1_{\left\{\left|X_{r}^{1, n}-X_{r}^{2, n}\right| \leq \frac{1}{k}\right\}} 1_{\left\{\left|X_{r}^{1, n}\right| \leq \sqrt{k}\right\}} d r\right) \\
& +\limsup _{n \rightarrow+\infty} \int_{s}^{t} P\left(\left|X_{r}^{1, n}\right| \geq \sqrt{k}\right) d r .
\end{aligned}
$$

Since $P\left(\left|X_{r}^{1, n}\right| \geq \sqrt{k}\right) \leq P\left(\left|B_{r}^{1}\right| \geq \frac{\sqrt{k}-r}{2}\right)+P\left(\left|X_{0}^{1}\right| \geq \frac{\sqrt{k}-r}{2}\right)$, the second term of the right-hand-side of (2.12) has a limit equal to 0 when $k \rightarrow+\infty$. To prove that the same is true for the first term, we bound the $L^{2}$ norm of the density of $\mathcal{L}\left(\left(X_{r}^{1, n}, X_{r}^{2, n}\right)\right)(r>0)$ uniformly in $n$. Like in the beginning of the proof of Lemma 2.3, we obtain an estimate similar to (2.6):

$$
\forall f \in L^{2}\left(\mathbb{R}^{2}\right), \forall n \geq 2, \forall r>0, \mathbb{E}\left(f\left(X_{r}^{1, n}, X_{r}^{2, n}\right)\right) \leq \frac{1}{\sqrt{2 \pi r}} \exp (r)\|f\|_{L^{2}} .
$$

Hence $\forall n \geq 2, \mathbb{E}\left(\int_{s}^{t} 1_{\left\{\left|X_{r}^{1, n}-X_{r}^{2, n}\right| \leq \frac{1}{k}\right\}} 1_{\left\{\left|X_{r}^{1, n}\right| \leq \sqrt{k}\right\}} d r\right) \leq \frac{C}{k^{\frac{1}{4}}}$ which implies

$$
\lim _{k \rightarrow+\infty} \limsup _{n \rightarrow+\infty} \mathbb{E}\left(\int_{s}^{t} 1_{\left\{\left|X_{r}^{1, n}-X_{r}^{2, n}\right| \leq \frac{1}{k}\right\}} 1_{\left\{\left|X_{r}^{1, n}\right| \leq \sqrt{k}\right\}} d r\right)=0 .
$$

By (2.11) and (2.12) we get $\lim _{k \rightarrow+\infty} \lim \sup _{n \rightarrow+\infty} \mathbb{E}\left(\left(G_{k}-G_{n}\right)^{2}\left(\mu^{n}\right)\right)=0$.

As we have proved that each term of the right-hand-side of (2.10) is equal to $0, \mathbb{E}^{\pi \infty}\left(G^{2}(\nu)\right)=0$. Restricting $\phi, g, s_{1}, \ldots, s_{p}, s, t$ to countable subsets then taking limits by Lebesgue's theorem, we obtain that $\pi_{\infty}$ a.s., $\nu$ solves 
the martingale problem

$$
\left\{\begin{array}{l}
\nu_{0}=m \otimes m \text { and } \forall \phi \in C_{b}^{2}\left(\mathbb{R}^{2}\right), \\
\phi\left(X_{t}, Y_{t}\right)-\phi\left(X_{0}, Y_{0}\right)-\int_{0}^{t} \frac{1}{2}\left(\frac{\partial^{2} \phi}{\partial x^{2}}+2 \frac{\partial^{2} \phi}{\partial x \partial y}+\frac{\partial^{2} \phi}{\partial y^{2}}\right)\left(X_{s}, Y_{s}\right) d s \\
\quad-\int_{0}^{t}\left(\frac{\partial \phi}{\partial x}\left(X_{s}, Y_{s}\right)\left(H * \bar{\nu}_{s}\left(X_{s}\right)\right)^{q}+\frac{\partial \phi}{\partial y}\left(X_{s}, Y_{s}\right)\left(H * P_{s}\left(Y_{s}\right)\right)^{q}\right) d s \\
\quad \text { is a } \nu \text {-martingale. }
\end{array}\right.
$$

Let us now suppose that $\nu$ is a solution of this problem.

Choosing $\phi(x, y)=\psi(x)$ with $\psi \in C_{b}^{2}(\mathbb{R})$, we check that $\nu \circ X^{-1}$ solves the nonlinear martingale problem starting at $m$. By uniqueness for this problem, $\nu \circ X^{-1}=P$ and $\bar{\nu}_{s}=P_{s}$. Moreover, it is easy to see that

$\beta_{t}^{1}=X_{t}-X_{0}-\int_{0}^{t}\left(H * P_{s}\left(X_{s}\right)\right)^{q} d s$ and $\beta_{t}^{2}=Y_{t}-Y_{0}-\int_{0}^{t}\left(H * P_{s}\left(Y_{s}\right)\right)^{q} d s$

are $\nu$-Brownian motions and next that $\beta^{1}=\beta^{2}$. As $\nu$ a.s., $Y_{0}=X_{0}$, by trajectorial uniqueness for the stochastic differential equation satisfied by both $X$ and $Y, \nu$ a.s., $X=Y$. Hence $\nu=\hat{P}$.

We conclude that $\pi_{\infty}=\delta_{\hat{P}}$ which puts an end to the proof.

\section{The Moderate MARTINGALE PROBLEM}

\subsection{Existence and Uniqueness}

Let $F$ be a measurable $\mathbb{R}^{d}$ valued function on $[0,+\infty) \times \mathbb{R}^{d} \times \mathbb{R}$ bounded by $M_{F}$ which satisfies the following Lipschitz continuity property

$$
\forall s \geq 0, \forall x \in \mathbb{R}^{d}, \forall y, y^{\prime} \in \mathbb{R},\left|y F(s, x, y)-y^{\prime} F\left(s, x, y^{\prime}\right)\right| \leq K_{F}\left|y-y^{\prime}\right| .
$$

Definition 3.1. Let $m \in \mathcal{P}\left(\mathbb{R}^{d}\right)$. We say that $P \in \tilde{\mathcal{P}}(\Omega)$ solves the nonlinear martingale problem (MP2) starting at $m$ if $P_{0}=m$ and for any $\phi \in C_{b}^{2}\left(\mathbb{R}^{d}\right)$

$$
\phi\left(X_{t}\right)-\phi\left(X_{0}\right)-\int_{0}^{t}\left(\frac{1}{2} \Delta \phi\left(X_{s}\right)+F\left(s, X_{s}, p\left(s, X_{s}\right)\right) \cdot \nabla \phi\left(X_{s}\right)\right) d s
$$

is a $P$-martingale where $p$ is a measurable version of the densities for $P$.

This definition does not depend on the choice of the measurable version. Indeed, if $p^{\prime}(s, x)$ is another such version then $P$ a.s., $\forall t \geq 0$,

$$
\int_{0}^{t} F\left(s, X_{s}, p\left(s, X_{s}\right)\right) \cdot \nabla \phi\left(X_{s}\right) d s=\int_{0}^{t} F\left(s, X_{s}, p^{\prime}\left(s, X_{s}\right)\right) . \nabla \phi\left(X_{s}\right) d s .
$$

Theorem 3.2. For any $m \in \mathcal{P}\left(\mathbb{R}^{d}\right)$, the nonlinear problem (MP2) admits a unique solution $P$ starting at $m$.

Proof. Uniqueness. It is an easy consequence of the Lipschitz continuity assumption made on $F$. The proof was given by Méléard et al (1987).

Let $P$ and $Q$ be two solutions of (MP2) starting at $m$ and $p(s, x), q(s, x)$ denote measurable versions of the densities for $P$ and $Q$. Using equation 
(2.2) given by Lemma 2.3, inequality (1.1) and the Lipschitz continuity property satisfied by $F$, we get

$$
\|p(t, .)-q(t, .)\|_{L^{1}} \leq \sqrt{d} K_{F} \int_{0}^{t} \frac{\|p(s, .)-q(s, .)\|_{L^{1}}}{\sqrt{t-s}} d s .
$$

By Gronwall's lemma, we conclude that for any $t>0,\|p(t, .)-q(t, .)\|_{L^{1}}=0$. Hence both $P$ and $Q$ solve the martingale problem in which the nonlinearity in (3.1) is replaced by $q\left(s, X_{s}\right)$. By uniqueness for this problem, $P=Q$.

Existence. In the sequel, if $I$ is a real interval and $v \in C\left(I, L^{1}\left(\mathbb{R}^{d}\right)\right)$ let $v(t, x)$ denote a measurable function on $I \times \mathbb{R}^{d}$ such that for any $t \in I$ the class of $v(t,$.$) in L^{1}\left(\mathbb{R}^{d}\right)$ is $v(t)$.

Let $T>0$ and $A_{T}=\left\{v \in C\left((0, T], L^{1}\left(\mathbb{R}^{d}\right)\right) ; \sup _{t \in(0, T]}\|v(t)\|_{L^{1}}<+\infty\right\}$. For the metric $D\left(v, v^{\prime}\right)=\sup _{t \in(0, T]}\left\|v(t)-v^{\prime}(t)\right\|_{L^{1}}, A_{T}$ is complete.

Let $m \in \mathcal{P}\left(\mathbb{R}^{d}\right)$. For $v \in A_{T}$, we set

$$
\forall t \in(0, T], \psi_{m}(v)(t)=G_{t} * m-\sum_{i=1}^{d} \int_{0}^{t} \frac{\partial G_{t-s}}{\partial x_{i}} *\left(v(s, .) F_{i}(s, ., v(s, .))\right) d s .
$$

By the continuity of the map $t \rightarrow G_{t} \in L^{1}\left(\mathbb{R}^{d}\right), t \rightarrow G_{t} * m \in L^{1}\left(\mathbb{R}^{d}\right)$ is continuous for $t>0$. Since

$$
\sup _{s \in(0, T]}\left\|v(s, .) F_{i}(s, ., v(s, .))\right\|_{L^{1}} \leq \sqrt{d} M_{F} \sup _{s \in(0, T]}\|v(s, .)\|_{L^{1}}<+\infty
$$

it is quite easy to deduce that $\psi_{m}(v) \in A_{T}$. Let us find $T$ such that $\psi_{m}$ is a contraction. For $v, v^{\prime} \in A_{T}$ and $t \in(0, T]$, we get an estimate similar to $(3.2)$

$$
\begin{aligned}
\left\|\psi_{m}(v)(t)-\psi_{m}\left(v^{\prime}\right)(t)\right\|_{L^{1}} & \leq \sqrt{d} K_{F} \int_{0}^{t} \frac{\left\|v(s)-v^{\prime}(s)\right\|_{L^{1}}}{\sqrt{t-s}} d s \\
& \leq 2 K_{F} \sqrt{d t} D\left(v, v^{\prime}\right) .
\end{aligned}
$$

Hence $D\left(\psi_{m}(v), \psi_{m}\left(v^{\prime}\right)\right) \leq 2 K_{F} \sqrt{d T} D\left(v, v^{\prime}\right)$. From now on, $T=\frac{1}{16 d K_{F}^{2}}$. By Picard's fixed-point theorem, $\psi_{m}$ admits a unique fixed-point in $A_{T}$. Let $t_{0} \geq 0$ and $f \in L^{1}\left(\mathbb{R}^{d}\right)$. For $v \in C\left([0, T], L^{1}\left(\mathbb{R}^{d}\right)\right)$ we define

$$
\tilde{\psi}_{t_{0}, f}(v)(t)=G_{t} * f-\sum_{i=1}^{d} \int_{0}^{t} \frac{\partial G_{t-s}}{\partial x_{i}} *\left(v(s, .) F_{i}\left(t_{0}+s, ., v(s, .)\right)\right) d s .
$$

The same estimates as above imply that $\tilde{\psi}_{t_{0}, f}$ admits a unique fixed-point in $C\left([0, T], L^{1}\left(\mathbb{R}^{d}\right)\right)$.

Let $v^{0}$ denote the fixed-point of $\psi_{m}$ in $A_{T}$. If $v^{n}$ is constructed, let $v^{n+1}$ be the fixed-point of $\tilde{\psi}_{(n+1) T, v^{n}(T)}$ in $C\left([0, T], L^{1}\left(\mathbb{R}^{d}\right)\right)$. We set $v(t)=v^{n}(t-n T)$ if $t \in(n T,(n+1) T]$. The map $v$ belongs to $C\left((0,+\infty), L^{1}\left(\mathbb{R}^{d}\right)\right)$ and satisfies

$$
\forall t_{0}>0, \sup _{t \in\left(0, t_{0}\right]}\|v(t)\|_{L^{1}}<+\infty .
$$


Let $t \in(0, T]$. We compute $v(T+t)$ thanks to Fubini's theorem.

$$
\begin{aligned}
v(T+t)=G_{t} * v(T) & -\sum_{i=1}^{d} \int_{0}^{t} \frac{\partial G_{t-s}}{\partial x_{i}} *\left(v(T+s, .) F_{i}(T+s, ., v(T+s, .))\right) d s \\
= & G_{t} *\left(G_{T} * m-\sum_{i=1}^{d} \int_{0}^{T} \frac{\partial G_{T-s}}{\partial x_{i}} *\left(v(s, .) F_{i}(s, ., v(s, .))\right) d s\right) \\
& \quad-\sum_{i=1}^{d} \int_{0}^{t} \frac{\partial G_{t-s}}{\partial x_{i}} *\left(v(T+s, .) F_{i}(T+s, ., v(T+s, .))\right) d s \\
= & G_{T+t} * m-\sum_{i=1}^{d} \int_{0}^{T+t} \frac{\partial G_{T+t-s}}{\partial x_{i}} *\left(v(s, .) F_{i}(s, ., v(s, .))\right) d s .
\end{aligned}
$$

By induction, we conclude that for any $t>0$,

$$
v(t, x)=G_{t} * m(x)-\sum_{i=1}^{d} \int_{0}^{t} \frac{\partial G_{t-s}}{\partial x_{i}} *\left(v(s, .) F_{i}(s, ., v(s, .))\right)(x) d s \text { a.s. }
$$

Let $P$ be the solution of the martingale problem in which the nonlinearity in (3.1) is replaced by $v\left(s, X_{s}\right)$. Equations (3.3), (3.4) and Lemma $2.3 \mathrm{imply}$ that $v(s, x)$ is a measurable version of the densities for $P$. Hence $P$ solves (MP2).

\subsection{Application}

Theorem 3.2 allows us to associate a probabilistic representation with some classical solutions of Burgers' equation. The initial conditions concerned are not distribution functions like in Proposition 2.4 but bounded probability densities.

We take up the approach of Oelschläger (1985) (pp. 306-307). Let $u_{0}$ be a probability density on $\mathbb{R}$ bounded by $M$. The Cole-Hopf transformation (Cole (1951), Hopf (1950))

$u(0, x)=u_{0}(x)$ and $u(t, x)=\frac{\int_{\mathbb{R}} G_{t}(x-y) \exp \left(-\int_{-\infty}^{y} u_{0}(z) d z\right) u_{0}(y) d y}{\int_{\mathbb{R}} G_{t}(x-y) \exp \left(-\int_{-\infty}^{y} u_{0}(z) d z\right) d y}$

provides a classical solution of Burgers' equation: $u \in C^{1,2}((0,+\infty) \times \mathbb{R})$ and

$$
\forall t>0, \forall x \in \mathbb{R}, \frac{\partial u}{\partial t}(t, x)=\frac{1}{2} \frac{\partial^{2} u}{\partial x^{2}}(t, x)-u(t, x) \frac{\partial u}{\partial x}(t, x) .
$$

It is easy to check that $\forall t>0, \forall x \in \mathbb{R},|u(t, x)| \leq M$. This boundedness property is essential for the sequel. We set $f(y)=\frac{1}{2}(0 \vee y \wedge M)$. The functions $f$ and $y \rightarrow y f(y)$ are respectively bounded and Lipschitz continuous. By Theorem 3.2, the martingale problem (MP2) corresponding to the particular choice $F(s, x, y)=f(y)$ admits a unique solution $P$ starting at $u_{0}(x) d x$. Let us prove that $u$ is a measurable version of the densities for $P$. Since 
clearly $\forall t \geq 0,\|u(t, .)\|_{L^{1}} \leq \epsilon$, according to the proof of uniqueness for (MP2) (Theorem 3.2), it is enough to establish

$$
\forall t>0, \forall x \in \mathbb{R}, u(t, x)=G_{t} * u_{0}(x)-\int_{0}^{t} \frac{\partial G_{t-s}}{\partial x} *(u(s, .) f(u(s, .)))(x) d s .
$$

Let $t>0, \phi$ be a $C^{1,2}$ function with compact support in $[0, t] \times \mathbb{R}$ and $\epsilon \in(0, t)$. As $\frac{\partial u}{\partial s}, \frac{\partial^{2} u}{\partial x^{2}}$ and $\frac{\partial}{\partial x}(u f(u))=u \frac{\partial u}{\partial x}$ are bounded on the intersection of the support of $\phi$ with $[\epsilon, t] \times \mathbb{R}$, using the integration by parts formula, Fubini's theorem and (3.5) we get

$$
\begin{aligned}
\int_{\mathbb{R}} u(t, x) \phi(t, x) d x & =\int_{\mathbb{R}} u(\epsilon, x) \phi(\epsilon, x) d x \\
& +\int_{(\epsilon, t] \times \mathbb{R}} u(s, x)\left(\frac{\partial \phi}{\partial s}+\frac{1}{2} \frac{\partial^{2} \phi}{\partial x^{2}}+f(u) \frac{\partial \phi}{\partial x}\right)(s, x) d x d s .
\end{aligned}
$$

We have $\lim _{s \rightarrow 0}\left\|u(s, .)-u_{0}\right\|_{L^{1}}=0$. Indeed for $U(x)=\exp \left(-\int_{-\infty}^{x} u_{0}(z) d z\right)$,

$$
\begin{aligned}
\left\|u(s, .)-u_{0}\right\|_{L^{1}} & \leq\left\|\frac{1}{G_{s} * U}\right\|_{L^{\infty}}\left\|G_{s} *\left(U u_{0}\right)-\left(G_{s} * U\right) u_{0}\right\|_{L^{1}} \\
& \leq e\left\|G_{s} *\left(U u_{0}\right)-U u_{0}\right\|_{L^{1}}+e\left\|\left(G_{s} * U-U\right) u_{0}\right\|_{L^{1}} .
\end{aligned}
$$

Since $U u_{0} \in L^{1}(\mathbb{R})$, the first term of the right hand side converges to 0 when $s \rightarrow 0$. The continuity and the boundedness of $U$ imply that $G_{s} * U$ is bounded uniformly in $s$ and converges pointwise to $U$. Hence, by Lebesgue's theorem, the second term also goes to 0 .

Thus $\lim _{s \rightarrow 0} \int_{\mathbb{R}} u(s, x) \phi(s, x) d x=\int_{\mathbb{R}} u_{0}(x) \phi(0, x) d x$ and taking the limit $\epsilon \rightarrow 0$ in $(3.7)$, we get

$$
\begin{aligned}
\int_{\mathbb{R}} u(t, x) \phi(t, x) d x & =\int_{\mathbb{R}} u_{0}(x) \phi(0, x) d x \\
& +\int_{(0, t] \times \mathbb{R}} u(s, x)\left(\frac{\partial \phi}{\partial s}+\frac{1}{2} \frac{\partial^{2} \phi}{\partial x^{2}}+f(u) \frac{\partial \phi}{\partial x}\right)(s, x) d x d s .
\end{aligned}
$$

By spatial truncation, this equation still holds if $\phi \in C_{b}^{1,2}([0, t] \times \mathbb{R})$. For the particular choice $\phi(s, x)=G_{t-s} * \psi(x)$ with $\psi C^{2}$ with compact support in $\mathbb{R}$, we conclude like in the proof of Lemma 2.3 that (3.6) holds. Therefore $u(t, x)$ is a measurable version of the densities for $P$ and $P$ provides a probabilistic representation of $u$.

\section{Extension of THE RESUlts to MARTingale PROBlEMS With \\ A NON-CONSTANT DIFFUSION COEFFICIENT}

Let $a$ be a Lipschitz continuous map on $\mathbb{R}^{d}$ with values in the set of symmetric non-negative $d \times d$ matrices such that

$$
\exists M_{a} \geq m_{a}>0, \forall x, y \in \mathbb{R}^{d}, m_{a}|y|^{2} \leq y^{*} a(x) y \leq M_{a}|y|^{2}
$$

and $L$ be the operator $L \phi(x)=\frac{1}{2} \sum_{i, j=1}^{d} a_{i, j}(x) \frac{\partial^{2} \phi}{\partial x_{i} \partial x_{j}}(x)$.

Let $\sigma$ denote the square-root of $a$. By the assumptions made on $a$, the map $x \rightarrow \sigma(x)$ is bounded and Lipschitz continuous. 
According to Friedman (1975) (pp. 139-150), there is a transition density $\Gamma_{s}(x, y), s>0, x, y \in \mathbb{R}^{d}$ associated with the time-homogeneous stochastic differential equation $d X_{t}=\sigma\left(X_{t}\right) d B_{t}$.

Moreover, for any $t>0$ and any continuous function $\psi$ with compact support in $\mathbb{R}^{d}$, the function $\phi(s, x)=\int_{\mathbb{R}^{d}} \Gamma_{t-s}(x, y) \psi(y) d y$ defined on $[0, t) \times \mathbb{R}^{d}$ satisfies

$$
\begin{aligned}
& \forall s \in[0, t), \forall x \in \mathbb{R}^{d}, L \phi(s, x)+\frac{\partial \phi}{\partial s}(s, x)=0, \\
& \forall x \in \mathbb{R}, \lim _{s \nearrow t} \phi(s, x)=\psi(x) .
\end{aligned}
$$

Lastly, for any $M>M_{a}$, there is a constant $C(t)$ such that,

$$
\begin{aligned}
& \forall s \in(0, t], \forall x, y \in \mathbb{R}^{d}, \Gamma_{s}(x, y) \leq \frac{C(t)}{s^{\frac{d}{2}}} \exp \left(-\frac{|x-y|^{2}}{2 M s}\right) \\
& \forall s \in(0, t], \forall x, y \in \mathbb{R}^{d}, \forall 1 \leq i \leq d,\left|\frac{\partial \Gamma_{s}(x, y)}{\partial x_{i}}\right| \leq \frac{C(t)}{s^{\frac{d+1}{2}}} \exp \left(-\frac{|x-y|^{2}}{2 M s}\right) .
\end{aligned}
$$

Integrating (4.3), we obtain the following estimate

$$
\forall t>0, \exists K(t)>0, \forall s \in(0, t], \forall x \in \mathbb{R}^{d}, \forall 1 \leq i \leq d,\left\|\frac{\partial \Gamma_{s}(x, y)}{\partial x_{i}}\right\|_{L_{y}^{1}} \leq \frac{K(t)}{\sqrt{s}} .
$$

THEOREM 4.1. $\forall m \in \mathcal{P}\left(\mathbb{R}^{d}\right)$, the martingale problem (MP1) (resp. (MP2)) in which $\frac{1}{2} \Delta \phi\left(X_{s}\right)$ is replaced by $L \phi\left(X_{s}\right)$ in (2.1) (resp. in (3.1)) admits a unique solution starting at $m$.

The proofs of Theorem 2.2 and Theorem 3.2 are based on Lemma 2.3. Therefore we explain how to adapt the conclusions and the proof of this lemma. As $\sigma$ is Lipschitz continuous and bounded, for any $m \in \mathcal{P}\left(\mathbb{R}^{d}\right)$, the martingale problem: $P_{0}=m$ and

$$
\forall \phi \in C_{b}^{2}\left(\mathbb{R}^{d}\right), \phi\left(X_{t}\right)-\phi\left(X_{0}\right)-\int_{0}^{t} L \phi\left(X_{s}\right) d s \text { is a } P \text {-martingale }
$$

admits a unique solution $P$. Moreover, by the existence of $\Gamma$, for $t>0$, $P_{t}$ has a density equal to $\int_{\mathbb{R}^{d}} \Gamma_{t}(x, y) m(d x)$. For $g$ like in Lemma 2.3, by Girsanov's theorem, as $\sigma^{-1} g$ is bounded, the martingale problem with $L \phi\left(X_{s}\right)+g\left(s, X_{s}\right) \cdot \nabla \phi\left(X_{s}\right)$ replacing $L \phi\left(X_{s}\right)$ admits a unique solution and this solution belongs to $\tilde{\mathcal{P}}(\Omega)$. Let $p(s, x)$ be a measurable version of the densities for the solution. If $\psi$ is a continuous function with compact support on $\mathbb{R}^{d}$ and $\phi(s, x)=\int_{\mathbb{R}^{d}} \Gamma_{t-s}(x, y) \psi(y) d y$, by the uniform continuity of $\psi$ and (4.2), the convergence of $\phi(s, x)$ to $\psi(x)$ in (4.1) is uniform in $x \in \mathbb{R}^{d}$. By (4.4), we upper-bound $\nabla \phi(s, x)$. These two remarks allow to transpose the proof of (2.2) and obtain that for any $t>0$,

$$
p(t, y)=\int_{\mathbb{R}^{d}} \Gamma_{t}(x, y) m(d x)+\int_{0}^{t} \int_{\mathbb{R}^{d}} \nabla_{x} \Gamma_{t-s}(x, y) . g(s, x) p(s, x) d x d s \text { a.s. }
$$

With this equation and (4.4) instead of (2.2) and (1.1), we easily adapt the proofs of Theorem 2.2 and Theorem 3.2 . 


\section{ACKNOWLEDGMENTS}

I would like to thank Professor Sylvie Méléard for introducing me to this topic and helping me in this work.

\section{REFERENCES}

Bossy, M. and Talay, D. (1996). Convergence Rate for the Approximation of the limit law of weakly interacting particles: Application to the Burgers Equation. Ann. A ppl. Prob. 6 818-861.

ColE, J. D. (1951). On a quasi-linear parabolic equation occuring in aerodynamics. Quart. Appl. Math. 9 225-236.

Friedman, A. (1975). Stochastic Differential Equations and Applications. Academic Press.

Graham, C. (1992). Nonlinear diffusions with jumps. Ann. Inst. Henri Poincaré. 28 393-402.

Hopf, E. (1950). The partial differential equation $u_{t}+u u_{x}=\mu u_{x x}$. Comm. Pure Appl. Math. 3 201-230.

Karatzas, I. and Shreve, S. E. (1988). Brownian Motion and Stochastic Calculus. Springer-Verlag.

MÉlÉARD, S. and RoELly-CoppoletTA, S. (1987). A propagation of chaos result for a system of particles with moderate interaction. Stochastic Processes and their Application. 26 317-332.

Meyer, P. A. (1966). Probabilités et Potentiel. Hermann.

OELSCHL ÄGER, K. (1985). A law of large numbers for moderately interacting diffusion processes. Z. Wahrsch. Verw. Geb. $69279-322$.

Sznitman, A. S. (1991). Topics in propagation of chaos. École d'été de probabilités de Saint-Flour XIX - 1989. Lect. Notes in Math. 1464. Springer-Verlag.

Cermics-enPC, 6-8 avenue Blaise Pascal, Cité Descartes, Champs-surMarne, 77455 Marne la Vallée Cedex 2. E-mail: jourdainđcermics.enpc.fr 